PREPARED FOR THE U.S. DEPARTMENT OF ENERGY, UNDER CONTRACT DE-AC02-76CH03073

PPPL-3596

PPPL-3596

UC-70

Stochastic lon Heating at the Magnetopause due to Kinetic Alfvén Waves

by

Jay R. Johnson and C.Z. Cheng

August 2001

M

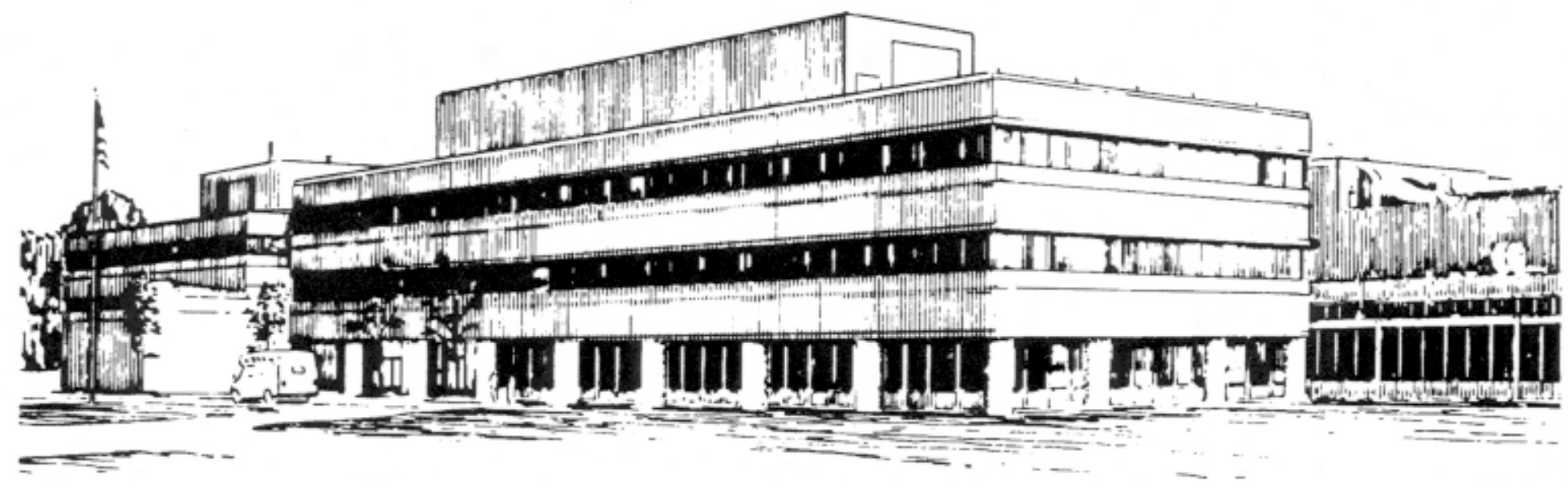

PRINCETON PLASMA PHYSICS LABORATORY PRINCETON UNIVERSITY, PRINCETON, NEW JERSEY 


\section{PPPL Reports Disclaimer}

This report was prepared as an account of work sponsored by an agency of the United States Government. Neither the United States Government nor any agency thereof, nor any of their employees, makes any warranty, express or implied, or assumes any legal liability or responsibility for the accuracy, completeness, or usefulness of any information, apparatus, product, or process disclosed, or represents that its use would not infringe privately owned rights. Reference herein to any specific commercial product, process, or service by trade name, trademark, manufacturer, or otherwise, does not necessarily constitute or imply its endorsement, recommendation, or favoring by the United States Government or any agency thereof. The views and opinions of authors expressed herein do not necessarily state or reflect those of the United States Government or any agency thereof.

\section{Availability}

This report is posted on the U.S. Department of Energy's Princeton Plasma Physics Laboratory Publications and Reports web site in Calendar Year 2001. The home page for PPPL Reports and Publications is: http://www.pppl.gov/pub_report/

DOE and DOE Contractors can obtain copies of this report from:

U.S. Department of Energy

Office of Scientific and Technical Information

DOE Technical Information Services (DTIS)

P.O. Box 62

Oak Ridge, TN 37831

Telephone: (865) 576-8401

Fax: (865) 576-5728

Email: reports@adonis.osti.gov

This report is available to the general public from:

National Technical Information Service

U.S. Department of Commerce

5285 Port Royal Road

Springfield, VA 22161

Telephone: 1-800-553-6847 or

(703) 605-6000

Fax: (703) 321-8547

Internet: http://www.ntis.gov/ordering.htm 


\title{
Stochastic ion heating at the magnetopause due to kinetic Alfvén waves
}

\author{
Jay R. Johnson and C. Z. Cheng \\ Princeton University, Plasma Physics Laboratory, Princeton, NJ
}

\begin{abstract}
The magnetopause and boundary layer are typically characterized by large amplitude transverse wave activity with freqency below the ion cyclotron frequency. The signatures of the transverse waves suggest that they are kinetic Alfvén waves with wavelength on the order of the ion gyroradius [Johnson and Cheng, 1997a; Johnson et al., 2001]. We investigate ion motion in the presence of large amplitude kinetic Alfvén waves with wavelength the order of $\rho_{i}$ and demonstrate that for sufficiently large wave amplitude $\left(\delta B_{\perp} / B_{0}>0.05\right)$ the particle orbits become stochastic. As a result, low energy particles in the core of the ion distribution can migrate to higher energy through the stochastic sea leading to an increase in $T_{\perp}$ and a broadening of the distribution. This process can explain transverse ion energization and formation of conics which have been observed in the low-latitude boundary layer.
\end{abstract}

\section{Introduction}

Large amplitude transverse wave activity accompanies nearly every magnetopause crossing [Perraut et al., 1979; Rezeau et al., 1993; Song et al., 1993c; Song, 1994; Phan and Paschmann, 1996; Johnson et al., 2001]. The wave spectrum is dominated by ULF waves with frequencies below $500 \mathrm{mHz}$ (less than the ion cyclotron frequency). There is growing evidence that those waves are kinetic Alfvén waves which result from mode conversion in the steep gradient of Alfvén velocity at the magnetopause [Johnson and Cheng, 1997a] or through nonlinear decay of mirror modes generated in the magnetosheath [Wu et al., 2001]. Often the observed wave amplitudes at the magnetopause can be significantly large compared with the background magnetic field, $B_{0}$. It is not uncommon for $\delta B_{\perp} / B_{0}$ to be the order of 0.2 or even larger [Rezeau et al., 1993; Song et al., 1993c, 1993b]. Moreover, in the cusp, it is likely that $\delta B_{\perp} / B_{0} \sim 1$ because of the weak background field.

The magnetopause provides a boundary between magnetosheath plasma and magnetospheric plasma. Plasma that leaks into the boundary layer is often found to have distinctive particle distributions indicative of acceleration processes. For example, electron distributions in the boundary layer are often found to be accelerated in the direction parallel to the magnetic field. These distributions have been identified as signatures of reconnection events, but the process which accelerates the electrons is not well known. It was suggested that electrons could also be accelerated by kinetic Alfvén waves [Lee et al., 1994] which preferentially heat electrons in the parallel direction due to the parallel electric field. Thermal electrons trapped in the wave potential would be heated leading to a slight increase in the parallel direction consistent with observations.

On the other hand, ions in the sheath transition layer and boundary layers often exhibit significant anisotropy with $T_{\perp}>T_{\|}$[Anderson et al., 1991; Song et al., 1993a]. Moreover, Wilber et al. [2001] have reported unusual low energy ion distribution components in the low-latitude boundary layer with pitch angles intermediate between 0 and 90 degrees observed by WIND/3DP during equatorial passes. The particles appeared to have undergone adiabatic streaming from a stronger magnetic field region with heating occuring near the magnetopause as deduced from the mirror ratios. The low energy ions appear to have been heated perpendicular to the magnetic field and in some events the core of the distribution appears to be flattened. The ion distributions presented by Song et al. [1993a] also illustrate that in the inner 
boundary layer the slope of the low energy component of the ion distribution function is flattened compared with the magnetosheath distribution suggestive that a physical process may be heating the low energy core of the distribution to higher energies.

In a recent study Johnson and Cheng [1997a] showed that when the kinetic Alfvén wave amplitude is sufficiently large, particle orbits can become stochastic leading to significant transport and particle heating. In this paper we will concentrate on particle heating. To study ion heating in the presence of a kinetic Alfvén wave, we will prescribe the electromagnetic fields consistent with the kinetic Alfvén waves. We investigate the particle motion in those prescribed fields as a function of the wave amplitude. The study will consist of a sequence of Poincaré sections taken at different wave amplitude which demonstrate the onset of stochasticity. The results will demonstrate: (1) stochastic ion heating can result through nonlinear coupling between low frequency waves and cyclotron motion, (2) ions can be heated transverse to the magnetic field leading to temperature anisotropy $\left(T_{\perp}>T_{\|}\right)$as observed at the magnetopause [Wilber et al., 2001], and (3) the stochastic process will deplete the core of the ion distribution function leading to a flattened core of the distribution function similar to the observations of [Wilber et al., 2001; Song et al., 1993a]

\section{Kinetic Alfvén Waves and Ion Heating}

The kinetic Alfvén wave is well described by three scalar quantities- $\phi, A_{\|}$, and $\delta B_{\|}$[Cheng and Johnson, 1999]. The fields associated with the kinetic Alfvén wave are obtained through Maxwell's equations given in Gaussian units.

$$
\begin{gathered}
\mathbf{E}=-\nabla \phi-\frac{1}{c} \frac{\partial A_{\|} \mathbf{b}}{\partial t} \\
\mathbf{B}=\mathbf{B}_{\mathbf{0}}(\mathbf{x})+\delta \mathbf{B} \approx\left(B_{0}(x)+\delta B_{\|}\right) \mathbf{b}-\mathbf{b} \times \nabla A_{\|}
\end{gathered}
$$

where $\mathbf{b}$ is the unit vector in the direction of the magnetic field. The vector potential is related to the electrostatic potential by introduction of a secondary potential, $\psi$ defined by

$$
E_{\|}=-\nabla \psi=-\nabla_{\|} \phi-\frac{1}{c} \frac{\partial A_{\|}}{\partial t}
$$

The fields are obtained by solving the set of equations described in Cheng and Johnson [1999] for the prescribed background magnetic field. If the background field is uniform, the kinetic Alfvén wave is a simple sinusoidal with $\phi=\phi_{0} \cos (\mathbf{k} \cdot \mathbf{x}-\omega t), \psi \approx$ $-\left(T_{e} / T_{i}\right) k_{\perp}^{2} \rho_{i}^{2} \phi /\left(1+k_{\perp}^{2} \rho_{i}^{2}\right), A_{\|}=\left(k_{\|} / \omega\right)(\phi-\psi)$. The wave satisfies the approximate dispersion relation

$$
\omega^{2}=k_{\|}^{2} V_{A}^{2}\left(1+\left(1+\frac{T_{e}}{T_{i}}\right) k_{\perp}^{2} \rho_{i}^{2}\right)
$$

where we ignore damping and take a Padé approximation for the Bessel function as described in Cheng and Johnson [1999]. The kinetic Alfvén wave is incompressible at low $\beta$ so that $\delta B_{\|} \approx 0$, but at $\beta \sim 1$ and short wavelength, $\delta B_{\|}$may also included as prescribed in Cheng and Johnson [1999]. For the chosen parameters $\left(k_{\perp} \rho_{i}=3, k_{\|} \rho_{i}=0.05\right.$, and $\left.\beta=1\right)$, $\left|\delta B_{\|}\right| \sim 0.55\left|\delta B_{\perp}\right|$ and is $90^{\circ}$ out of phase.

Particle orbits are determined by the equation of motion

$$
m_{i} \frac{d^{2} \mathbf{r}}{d t}=q_{i}\left(\mathbf{E}+\frac{\mathbf{v}}{c} \times \mathbf{B}\right)
$$

which we normalize to

$$
\ddot{\mathbf{X}}=-\tilde{\nabla} \Phi+\dot{\mathbf{X}} \times\left(\Delta \hat{\mathbf{b}}+\frac{\delta \mathbf{B}}{\tilde{B}_{0}}\right)
$$

where $\mathbf{X}=\mathbf{r} / \rho_{i}, \tau=\Omega t, \tilde{\nabla}=\rho_{i} \nabla, \Phi=q_{i} \phi / T_{i}$, with $\Omega=q_{i} \tilde{B}_{0} / m_{i} c, \rho_{i}=\sqrt{T_{i} / m_{i}} / \Omega, \Delta=\left|B_{0}(x) / \tilde{B}_{0}\right|$ and $\dot{\mathbf{X}} \equiv d \mathbf{X} / d \tau$, and $\tilde{B}_{0}$ is a characteristic value of magnetic field.

To investigate the behavior of particles in the kinetic Alfvén waves, we plot Poincaré sections for particle orbits. This technique is standard and has been applied to electrostatic waves to understand plasma heating well above the cyclotron frequency [Karney and Bers, 1977], at near the cyclotron frequency [Hsu et al., 1979]. Points on Poincaré sections are plotted at constant particle gyrophase, $\gamma$, with the requirement that $\dot{\gamma}<0$. In the absence of waves, this would correspond with one point per gyroperiod. At each crossing of the phase space plane defined by $\gamma=0$ where $\mathbf{k} \cdot \mathbf{b} \times \dot{\mathbf{X}}=k_{\perp} v_{\perp} \sin (\gamma)$, we plot the value of the magnetic moment, $\mu \equiv|\mathbf{b} \times \dot{\mathbf{X}}|^{2} / \Delta$, versus $\Psi=\mathbf{k} \cdot \mathbf{x}-\omega t$, taken modulo $2 \pi$. The choice of these variables is good for examining particle heating in the presence of the wave because the magnetic moment is an adiabatic invariant which follows well defined trajectories in phase space. The wave phase is an obvious choice because there is a direct correlation between the adiabatic particle motion and the wave amplitude.

To examine the onset of stochastic particle behavior in the presence of large amplitude kinetic Alfvén waves, we examine a sequence of Poincaré sections as 
a function of wave magnetic field amplitude. For simplicity, we assume a uniform background magnetic field. We specify $k_{\perp} \rho_{i}=3, \omega=\Omega_{i} / 5, \beta=1$, $T_{e} / T_{i}=0.2$ and $k_{\|} \rho_{i} \approx 0.05$ consistent with the kinetic Alfvén wave dispersion relation. The small perpendicular scales are consistent with typical kinetic Alfvén wave solutions at the magnetopause with wavelength the order of $100 \mathrm{~km} \approx 2 \rho_{i}$ [Johnson and Cheng, 1997a; Johnson et al., 2001].

For clarity of Poincaré section plots, we take the wave frequency equal to $1 / 5$ of the ion gyrofrequency. For smaller frequency similar physics leads to stochastic thresholds, but the formation of island chains composed of hundreds of islands cannot be as easily seen by eye. Interestingly, the stochastic threshold primarily depends on wave amplitude and is not strongly dependent on wave frequency in the range of interest. Note that because $k_{\perp} \rho_{i}$ is larger than 1 , the kinetic Alfvén wave does have a significant electrostatic component associated with ion Larmor radius effects.

The Poincaré section for $\delta B_{\perp} / B_{0}=0.006$ is shown in Figure 1. Particles are started with $\Psi=0$ with varying initial value of $\mu$ and $v_{\|}=0$. If there were no wave, the particles would simply gyrate with constant value of $\mu$. For small wave amplitude, $\mathbf{X}$ is nearly periodic in the gyrophase, so dependence of $\Psi$ on time is primarily through $-\omega t$. Because the wave frequency is $\Omega_{i} / 5, \Psi$ will approximately decrease by $2 \pi / 5$ each gyroperiod until it returns to the original phase (minus $2 \pi$ ). The Poincare section for a given particle would therefore reduce to five equally spaced points at constant $\mu$. However, with the addition of the wave, the particle gyration can be retarded or accelerated. Indeed, this can be seen from the equation of motion for constant background magnetic field

$\dot{\gamma}=\frac{\boldsymbol{\rho} \cdot \ddot{\mathbf{X}}}{\rho^{2}}=-\left(1+\mathbf{B}_{0} \cdot \delta \mathbf{B} / B_{0}^{2}\right)-\frac{\boldsymbol{\rho}}{\rho^{2}} \cdot \tilde{\nabla}_{\perp}\left(\Phi-A_{\|} \mathbf{b} \cdot \mathbf{X}\right)$

where $\boldsymbol{\rho}=\mathbf{b} \times \dot{\mathbf{X}}$ is the gyroradius vector. The gyromotion can be retarded/advanced by the perturbed electric and magnetic fields and clearly depends on gyroradius/magnetic moment.

In Figure 1, it is apparent that the for initial $\mu<0.41$ particles do not return to the same wave phase after a wave period (five gyroperiods), but have a small positive increment in phase $\Psi$. On the other hand, for initial $\mu>0.41$, it is apparent that the after a wave period, the phase, $\Psi$, of the particle will have a small negative increment. Near initial $\mu=0.41$, it is evident that there is a boundary where

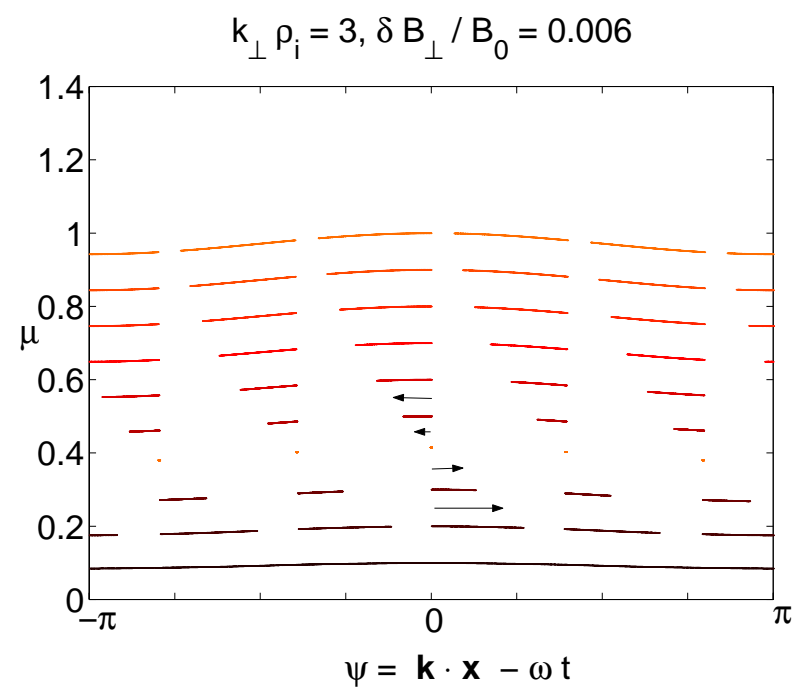

Figure 1. Poincaré section for small amplitude wave. Note that low energy particles are advanced in the phase of the wave while higher energy particles are retarded. Phase space islands with period five first emerge along the stationary phase trajectory.

the orbit is stationary and the Poincare section only consists of only five points. As the amplitude is increased these fixed points move to larger values of $\mu$ and nearby orbits circulate around the fixed points. Moreover, other boundaries appear across which the phase advances/retards, but with higher order periodicity. This phenomenon is illustrated in Figure 2 which shows the Poincaré section for $\delta B_{\perp} / B_{0}=0.05$. The period five island chain associated with the original transition boundary has moved to larger value of $\mu$ and is quite large in extent. Above that chain, the high energy particles do not show any structure related to the gyromotion and simply float up and down in the wave. Other islands chains have also appeared at lower energies. The obvious island periods evidently are in the sequence: $26,21,58,16,27$ and so forth. Islands in the period 16 and 21 chains have just begun to overlap.

At the lowest energies, a chain of five fingers (rather than islands) has appeared. The fingers have divided into ten subfingers. The central finger contains two islands.. The physical origin of the fingers is the increase of the $\mathbf{E} \times \mathbf{B}$ velocity of the wave. When low energy particles are subjected to large $\mathbf{E} \times \mathbf{B}$ motion, the gyrophase can reverse direction and the particles are trapped in the wave. As a result, the particles are not sampled during their gyromotion and the 


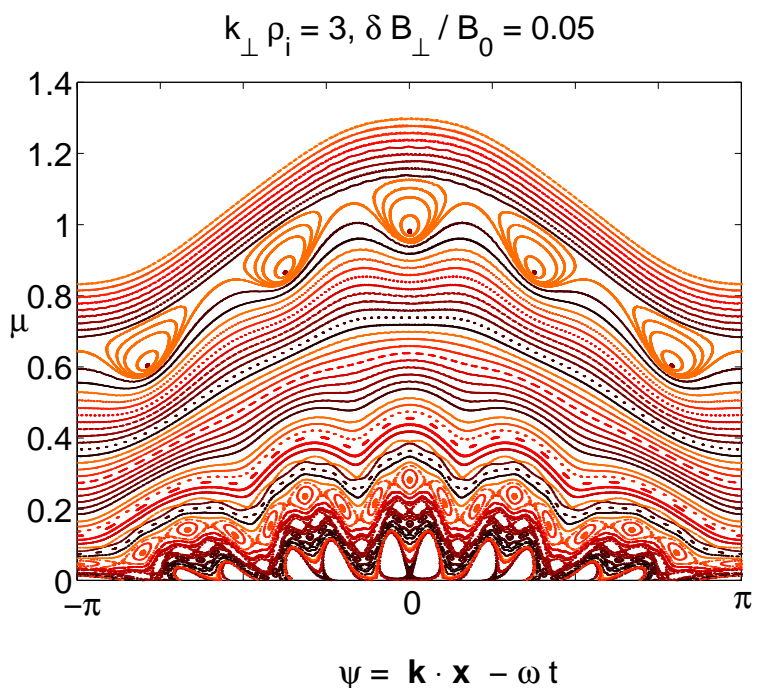

Figure 2. As the wave amplitude is increased, chains of phase space islands appear. The period 16 island chain has just begun to merge and a separatrix appears.

island is incomplete (that is, the low energy particles can skip one or more fingers during their gyromotion).

A slight increase in wave amplitude shown in Figure 3 allows the phase space islands to merge and regions of stochastic orbits appear. Island structures still remain embedded in the stochastic region, but now particle trajectories can wander through the stochastic sea to higher energies than previously accessible. Hence, the low energy part of the ion distribution can be effectively heated. Note that the ten finger structures have now moved into the stochastic sea and comprise a sequence of ten islands. A new five fingered structure has also begun to emerge from the low energy part of the phase space with regular orbits below the stochastic sea with a fixed point in the central island. The stationary orbit at $\Psi=0$ in the central finger is an elongated orbit which has period equal to that of the wave and can be considered to be in nonlinear resonance with the wave. Above the stochastic sea, particle trajectories lie on well defined curves or island chains and the particles are not heated. The large period five island chain remains intact and moves to higher energy. However, clear boundaries still confine heating to the lowest energy ions.

For $\delta B_{\perp} / B_{0} \sim 0.11$ as shown in Figure 4 nearly the entire low $\mu$ region becomes stochastic except for a few small islands that remain embedded in the

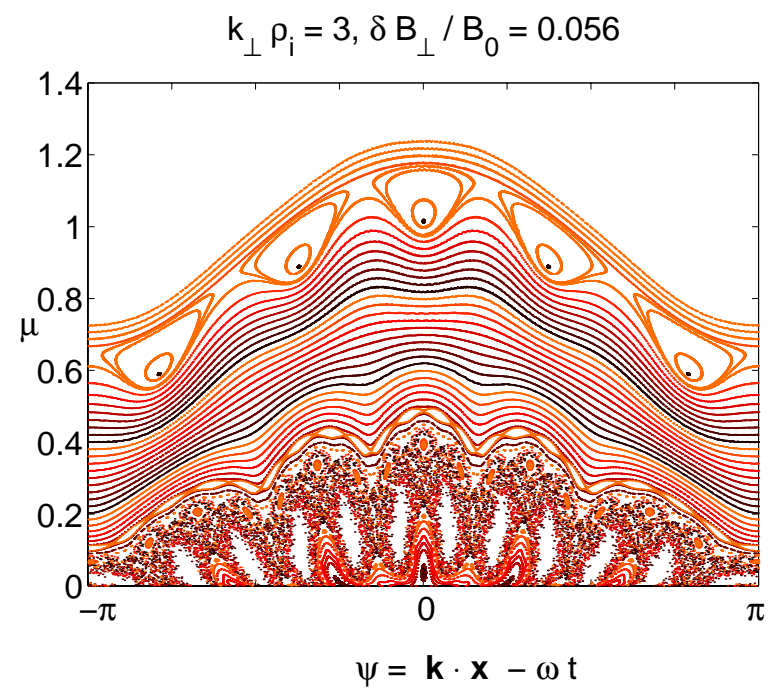

Figure 3. With a slight increase in wave amplitude, the low energy region of the Poincaré section becomes stochastic. Low energy particles can now wander through the stochastic sea to higher energies. Phase space islands remain embedded in the stochastic sea, but the entire region becomes stochastic for $\delta B / B_{0}=0.056$.

stochastic sea. The stochastic region is forced up against the set of period five islands which have themselves become stochastic. A clear boundary exists between the two stochastic regions and particles may not move across that boundary. Above the boundary, the period five island chain has also merged and become stochastic and other chains of islands have also appeared against the boundary between the lower stochastic region and the upper stochastic region. Chains of islands have also appeared inside the period five islands. However, the two regions are still separated and low energy particles cannot be energized much above the thermal speed. With a modest increase to $\delta B_{\perp} / B_{0}=0.14$ as shown in Figure 5 , the period five islands merge with the low energy stochastic region leaving a path for low energy particles to be heated well beyond the thermal energy. With further increase in the wave amplitude the stochastic regime pushes to larger $\mu$. For example, with $\delta B_{\perp} / B_{0} \sim 0.3$ particles are readily energized to $\mu \sim 4$.

\section{Discussion and Summary}

It is useful to discuss some of the properties of the waves and particle orbits. In this study we have fo- 


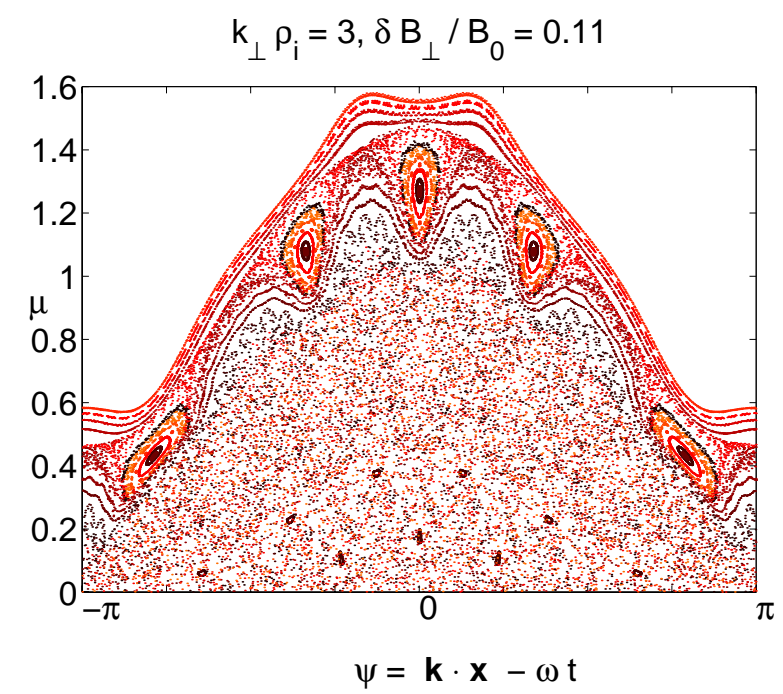

Figure 4. Both the period five island chain and the low energy region are stochastic, but they are separated by a boundary. Global stochasticity occurs when the two regions merge just above $\delta B_{\perp} / B_{0}=$ 0.11 .

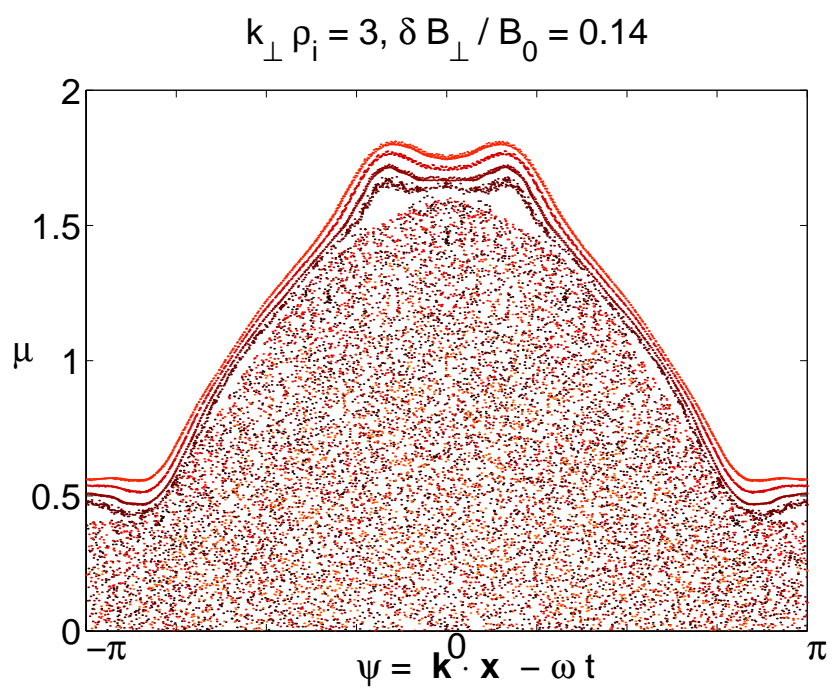

Figure 5. Global stochasticity for $\delta B_{\perp} / B_{0}=0.14$. Cold and/or thermal particles may wander to superthermal energies resulting in heating perpendicular to the magnetic field. . cused on particles with $v_{\|}<<v_{t i}$. However, for particles with larger $v_{\|}$heating is still primarily in the perpendicular direction. Because the parallel electric field is not large it does not seem to affect the parallel ion motion for non-resonant ions very much. Increases in the parallel velocity for low energy ions are primarily due to the magnetic field as $\dot{v}_{\|} \approx-\mathbf{v} \cdot \nabla_{\perp} A_{\|}$. Even for the stochastic orbits examined in this paper, the parallel velocity does not increase significantly (typically less than $1 \%$ of perpendicular energy). Although the parallel electric field could be significant for energizing particles which are moving with the parallel phase velocity of the wave, the parallel phase speed for kinetic Alfvén waves is significantly increased due to perpendicular dispersion so that wave trapping is more difficult even in a high $\beta$ plasma $\left(\omega / k_{\|} \sim 3 v_{t i}\right)$. Finally, the wave solution has a significant electrostatic component which is primarily responsible for the behavior of low energy particles, but the period five islands at larger values of $\mu$ appear to be determined by the perturbed magnetic field.

The Poincaré sections for waves with lower frequency are relevant to particle dynamics at the magnetopause because magnetopause wave spectra are dominated by waves with frequencies in the range 10$500 \mathrm{mHz} \approx 0.01-0.5 \Omega_{i}$. Poincaré sections are quite similar except more fingers appear at lower frequency and island chains can consist of hundreds of islands. Interestingly, the threshold appears to show no significant dependence on frequency for $\omega>0.01 \Omega_{i}$ although the threshold for onset of stochasticity does increase slightly for lower frequency. Similarly, heating rate and maximum energy of particles is slightly smaller for lower frequency. Irrational values of $\Omega_{i} / \omega$ also seem to produce ion heating with roughly the same threshold for onset of stochasticity.

Particle motion of low energy particles is dominated by the electrostatic electric field for large $k_{\perp} \rho_{i}$. As such, the stochastic threshold is mainly dependent on the value of $\Phi$ and $k_{\perp}$, and indeed, we have found that the stochastic regime occurs when $k_{\perp}^{2} \rho_{i}^{2} \Phi \sim 1$ without much sensitivity to frequency. For magnetopause conditions this would translate to modest electric field strength $-E \sim 5 \times$ $(\lambda / 100 \mathrm{~km})(B / 50 \mathrm{nT})^{2} \mathrm{mV} / \mathrm{m}$. Magnetic fields are approximately given by

$$
\frac{\delta B_{\perp}}{B_{0}} \sim \sqrt{\frac{\beta}{2}} \frac{\sqrt{1+\left(1+T_{e} / T_{i}\right) k_{\perp}^{2} \rho_{i}^{2}}}{k_{\perp} \rho_{i}\left(1+k_{\perp}^{2} \rho_{i}^{2}\right)}
$$

which is in rough agreement with our calculation $\left(\delta B / B_{0} \sim 0.1\right)$ and shows the dependence on wave- 
length and $\beta$.

These results have several implications relevant to magnetopause observations. First, transverse ion heating due to this process depends on wave amplitude. Below the wave threshold, there is no heating of the plasma. Slightly above the threshold, the core of the distribution is expected to flatten, but ions are not heated above the thermal velocity. For larger wave amplitude, ions can be heated above the thermal velocity. The heating process can occur rapidly over a time less than 30 cyclotron periods. Due to the brevity of this letter, we defer estimates of the stochastic threshold, more detailed description of the appearance of island chains, island overlap,, and discussion of the effect of background gradients (and the resulting particle transport) for a later publication.

Obviously, wave heating at the magnetopause is more complicated than this simple picture. This calculation is primarily meant to provide understanding of the nonlinear coupling between kinetic Alfvén waves and cyclotron motion and to provide a physical picture which gives qualitative understanding of resulting particle signatures. Most likely, there is a spectrum of waves that can participate in the heating process. Typically, the addition of a second wave or magnetic field rotation can reduce the threshold for stochasticity to occur and increase particle heating and transport beyond quasi-linear levels [Johnson and Cheng, 1997b].

In summary, we have examined ion motion in the presence of kinetic Alfvén waves prescribed for magnetopause conditions. We find that for typical Alfvén wave parameters, onset of stochasticity occurs when $\delta B / B_{0} \sim 0.05$, leading to full stochasticity of the thermal population when $\delta B / B_{0} \sim 0.1$ as first shown by Johnson and Cheng [1997b].

Acknowledgments This work is supported by the NSF grant ATM-9906142 and DoE Contract CGLNo. DE-AC02-76-CHO3073.

\section{References}

Anderson, B. J., S. A. Fuselier, and D. Murr, Electromagnetic ion cyclotron waves observed in the plasma depletion layer, Geophys. Res. Lett., 18, 1955, 1991.

Cheng, C. Z., and J. R. Johnson, A kinetic-fluid model, J. Geophys. Res., 104, 413-427, 1999.
Hsu, J. Y., K. Matsuda, M. S. Chu, and T. H. Jensen, Stochastic heating of a large-amplitude standing wave, Phys. Rev. Lett., 43, 203-206, 1979.

Johnson, J. R., and C. Z. Cheng, Kinetic Alfvén waves and plasma transport at the magnetopause, Geophys. Res. Lett., 24, 1423-1426, 1997a.

Johnson, J. R., and C. Z. Cheng, Plasma transport at the magnetopause due to low frequency mhd waves in a strongly sheared magnetic field, Eos Trans. $A G U$, 78(46), F591, Fall Meet. Suppl., 1997b.

Johnson, J. R., C. Z. Cheng, and P. Song, Signatures of mode conversion and kinetic Alfvén waves at the magnetopause, Geophys. Res. Lett., 28(2), 227-230, 2001.

Karney, C. F. F., and A. Bers, Stochastic ion heating by a perpendicularly propagating electrostatic wave, Phys. Rev. Lett., 39, 550, 1977.

Lee, L. C., J. R. Johnson, and Z. W. Ma, Kinetic Alfvén waves as a source of plasma transport at the dayside magnetopause, J. Geophys. Res., 99, 17405-17411, 1994.

Perraut, S., R. Gendrin, P. Robert, and A. Roux, Magnetic pulsations observed onboard GEOS 2 in the ULF ran ge during multiple magnetopause crossings, in Eur. Space Agency Spec. Publ., 148, pp. 113-122. 1979.

Phan, T. D., and G. Paschmann, Low-latitude dayside magnetopause and boundary layer for high magnetic shear 1. structure and motion, J. Geophys. Res., 101, 1996.

Rezeau, L., A. Roux, and C. T. Russell, Characterization of small-scale structures at the magnetopause from ISEE measurements, J. Geophys. Res., 98, 179, 1993.

Song, P., Observations of waves at the dayside magnetopause, in Solar Wind Source of Magnetospheric Ultra-Low-Frequency Waves, Geophysical Monograph Series, vol. 81, pp. 159-171. 1994.

Song, P., et al., Wave properties near the subsolar magnetopause for northward interplanetary magnetic field: Multiple instrument particle observations, J. Geophys. Res., 98, 11,319-11,337, 1993a. 
Song, P., C. T. Russell, and C. Y. Huang, Wave properties near the subsolar magnetopause: Pc 1 waves in the sheath transition layer, J. Geophys. Res., 98, 5907-5923, 1993b.

Song, P., C. T. Russell, R. J. Strangeway, J. R. Wygant, C. A. Cattell, R. J. Fitzenreiter, and R. R. Anderson, Wave properties near the subsolar magnetopause: Pc 3-4 energy coupling for northward interplanetary magnetic field, J. Geophys. Res., 98, 187-196, 1993c.

Wilber, M., G. K. Parks, and R. P. Lin, Conic-like ion components observed interior to the dusk magnetopause, AGU Chapman Conference, The Low Latitude Boundary Layer, New Orleans, LA, 2001.

Wu, B. H., J. M. Wang, and L. C. Lee, Generation of kinetic Alfvén waves by mirror instability, Geophys. Res. Lett., in press, 2001.

This preprint was prepared with the AGU LATEX macros v3.0. File GRL formatted 2001 August 1.

With the extension package ' $\mathrm{AGU}^{++}$', version 1.2 from $1995 / 01 / 12$ 


\section{External Distribution}

Plasma Research Laboratory, Australian National University, Australia

Professor I.R. J ones, Flinders University, Australia

Professor J oão Canalle, Instituto de Fisica DEQ/IF - UERJ , Brazil

Mr. Gerson O. Ludwig, Instituto Nacional de Pesquisas, Brazil

Dr. P.H. Sakanaka, Instituto Fisica, Brazil

The Librarian, Culham Laboratory, England

Library, R61, Rutherford Appleton Laboratory, England

Mrs. S.A. Hutchinson, JET Library, England

Professor M.N. Bussac, Ecole Polytechnique, France

Librarian, Max-Planck-Institut für Plasmaphysik, Germany

J olan Moldvai, Reports Library, MTA KFKI-ATKI, Hungary

Dr. P. Kaw, Institute for Plasma Research, India

Ms. P.J . Pathak, Librarian, Insitute for Plasma Research, India

Ms. Clelia De Palo, Associazione EURATOM-ENEA, I taly

Dr. G. Grosso, Instituto di Fisica del Plasma, Italy

Librarian, Naka Fusion Research Establishment, J AERI, J apan

Library, Plasma Physics Laboratory, Kyoto University, J apan

Research Information Center, National Institute for Fusion Science, J apan

Dr. O. Mitarai, Kyushu Tokai University, J apan

Library, Academia Sinica, Institute of Plasma Physics, People's Republic of China

Shih-Tung Tsai, Institute of Physics, Chinese Academy of Sciences, People's Republic of China

Dr. S. Mirnov, TRINITI, Troitsk, Russian Federation, Russia

Dr. V.S. Strelkov, Kurchatov Institute, Russian Federation, Russia

Professor Peter Lukac, Katedra Fyziky Plazmy MFF UK, Mlynska dolina F-2, Komenskeho Univerzita, SK-842 15 Bratislava, Slovakia

Dr. G.S. Lee, Korea Basic Science Institute, South Korea

Mr. Dennis Bruggink, Fusion Library, University of Wisconsin, USA

Institute for Plasma Research, University of Maryland, USA

Librarian, Fusion Energy Division, Oak Ridge National Laboratory, USA

Librarian, Institute of Fusion Studies, University of Texas, USA

Librarian, Magnetic Fusion Program, Lawrence Livermore National Laboratory, USA

Library, General Atomics, USA

Plasma Physics Group, Fusion Energy Research Program, University of California at San Diego, USA

Plasma Physics Library, Columbia University, USA

Alkesh Punjabi, Center for Fusion Research and Training, Hampton University, USA

Dr. W.M. Stacey, Fusion Research Center, Georgia Institute of Technology, USA

Dr. J ohn Willis, U.S. Department of Energy, Office of Fusion Energy Sciences, USA

Mr. Paul H. Wright, Indianapolis, Indiana, USA 
The Princeton Plasma Physics Laboratory is operated by Princeton University under contract with the U.S. Department of Energy.

\author{
Information Services \\ Princeton Plasma Physics Laboratory \\ P.O. Box 451 \\ Princeton, NJ 08543
}

Phone: 609-243-2750

Fax: 609-243-2751

e-mail: pppl_info@pppl.gov

Internet Address: http://www.pppl.gov 